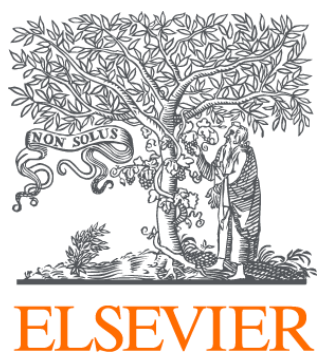

Since January 2020 Elsevier has created a COVID-19 resource centre with free information in English and Mandarin on the novel coronavirus COVID-

19. The COVID-19 resource centre is hosted on Elsevier Connect, the company's public news and information website.

Elsevier hereby grants permission to make all its COVID-19-related research that is available on the COVID-19 resource centre - including this research content - immediately available in PubMed Central and other publicly funded repositories, such as the WHO COVID database with rights for unrestricted research re-use and analyses in any form or by any means with acknowledgement of the original source. These permissions are granted for free by Elsevier for as long as the COVID-19 resource centre remains active. 


\section{Sharing methods for global health research: an assessment of methodology}

Trudie Lang, Kevin Marsh, Rosanna Peeling, Jeremy Farrar

\begin{abstract}
Background Setting up a clinical study can take more than 18 months and can cost millions of pounds. For many aspects of global health there is a dearth of research, yet evidence is urgently needed to address major challenges. Obtaining such data within a meaningful timeframe requires a fundamental change in the planning and setting up of research studies. Typically, protocols are developed in closed groups and often never shared. However, many operational processes in research are generic, irrespective of the disease or design. Thus, removal of duplication and sharing methods could speed up research. This research methodology project investigates whether researchers can be persuaded to share methods and if this will streamline research.
\end{abstract}

Methods The theory of communities of practice (in which distinct specialists working on a problem collaborate to solve it) guided this project. The Global Health Network was created to enable communities of practice to form to develop protocols, exchange research instruments, and share skills. We measured uptake, effect, and benefit of the Network through surveys, questionnaires, interviews with target audiences (research teams in low-income and middle-income countries), and analysis of digital access.

Findings Since 2011, more than 60000 researchers, nurses, technicians, and scientists from almost all low-income and middle-income countries have used the project to share research methods, knowledge, and processes. 21 specialised communities of practice have formed collaborations that are actively exchanging methods. More than 250 research guidance notes have been shared that provide specific guidance for various research methods-eg, an international respiratory disease network has released three open-access novel coronavirus protocols and groups working in maternal health are listing the samples they have stored with the invitation for others to collaborate. These qualitative and quantitative data show that these exchanges are highly beneficial for guiding and improving research operations, and that there is a willingness to share.

Interpretation Scientists compete for funding and publication and therefore sharing methods and knowledge can be perceived as disadvantageous. However, if use of protocols and methods developed by wide and open consensus can help to quickly gain high quality data, then better progress could be made for all and disease research could be changed to improve health.

Funding Bill \& Melinda Gates Foundation.

Contributors

TL, KM, RP, and JF designed the study and wrote the abstract.

Conflicts of interest

We declare that we have no conflicts of interest.

Acknowledgments

Members of the authorship groups did the interviews and analysed the questionnaires.
Published Online October 20, 2013 University of Oxford, Oxford UK (T Lang PhD); Kemri-Wellcome Programme, Kilifi, Kenya (K Marsh MD); London School Hygiene and Tropical Medicine, London, UK (R Peeling PhD); and Oxford Clinical Research Unit, Hospital for Tropical Disease, Ho Chi Min City, Vietnam (J Farrar MRCP)

Correspondence to:

DrTrudie Lang, University of Oxford, Oxford OX3 7LJ, UK trudie.lang@ndm.ox.ac.uk

For the Global Health Network see http://www.

theglobalhealthnetwork.org 